This item was submitted to Loughborough's Research Repository by the author.

Items in Figshare are protected by copyright, with all rights reserved, unless otherwise indicated.

\title{
Collective excitations and screening properties of a condensed charged
} Bose gas

PLEASE CITE THE PUBLISHED VERSION

PUBLISHER

() American Physical Society

VERSION

VoR (Version of Record)

LICENCE

CC BY-NC-ND 4.0

\section{REPOSITORY RECORD}

Alexandrov, A.S., and W.H. Beere. 2019. "Collective Excitations and Screening Properties of a Condensed Charged Bose Gas". figshare. https://hdl.handle.net/2134/4282. 
This item was submitted to Loughborough's Institutional Repository (https://dspace.lboro.ac.uk/) by the author and is made available under the following Creative Commons Licence conditions.

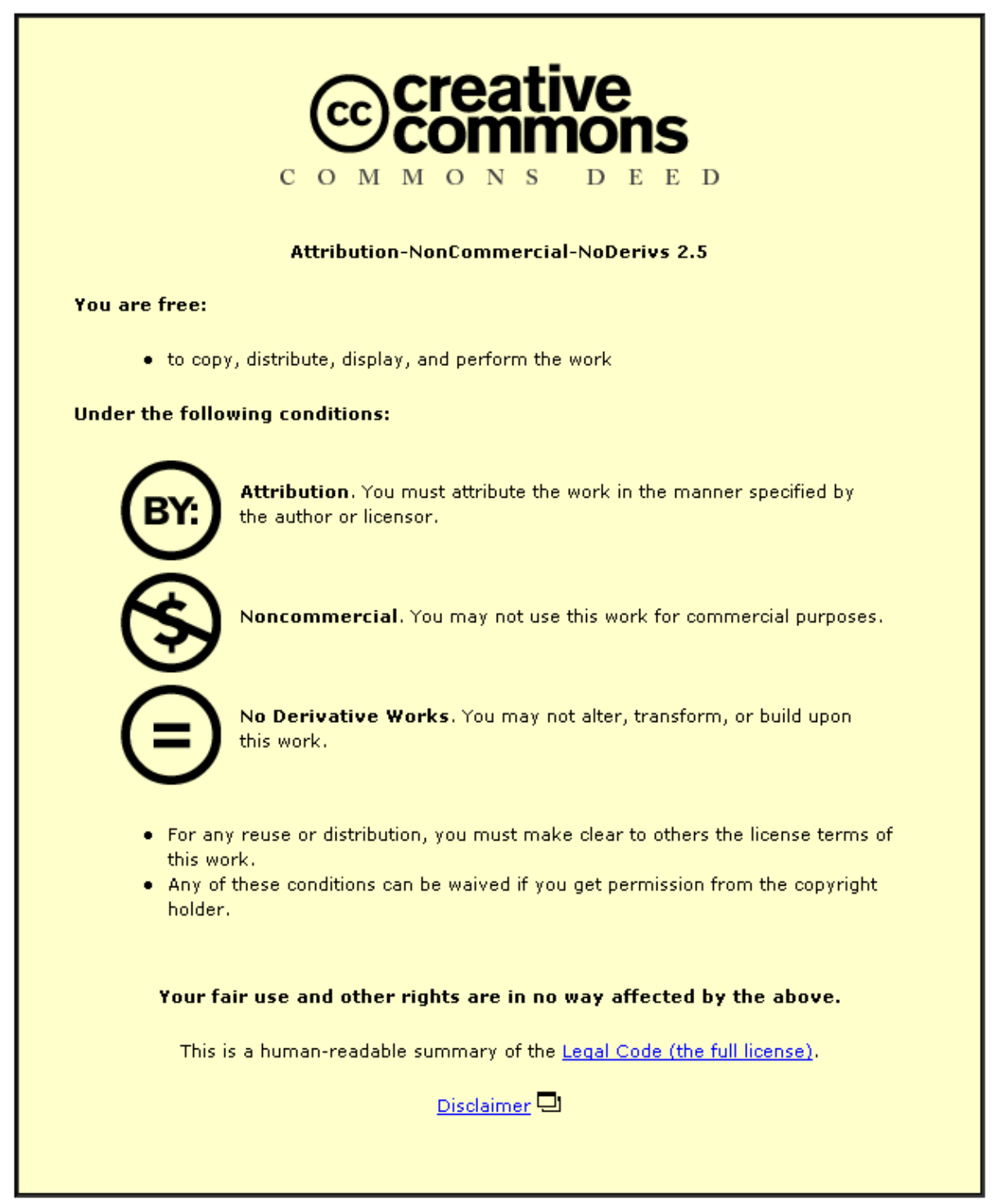

For the full text of this licence, please go to: http://creativecommons.org/licenses/by-nc-nd/2.5/ 


\title{
Collective excitations and screening properties of a condensed charged Bose gas
}

\author{
A. S. Alexandrov and W. H. Beere \\ Interdisciplinary Research Center in Superconductivity, University of Cambridge, Madingley Road, Cambridge, \\ CB3 OHE, United Kingdom
}

(Received 8 March 1994; revised manuscript received 15 November 1994)

\begin{abstract}
The Bogoliubov-de Gennes (BdG) type equations are derived for the Coulomb Bose gas describing the electrodynamics of charged bosons. The gauge invariant dielectric response function and collective excitations of a charged Bose gas at $T=0$ are found. We find a plasmon branch which is the same as the single-particle Bogoliubov spectrum in agreement with the textbook result. The superconducting properties of the charged Bose gas are verified. A gauge-invariant formalism for calculating the response of the supracondensate particles is proposed.
\end{abstract}

\section{INTRODUCTION}

A charged Bose gas has been studied by several authors and recently became of particular interest in the context of some theories of high-temperature superconductivity. ${ }^{1}$ The one-particle excitation spectrum at $T=0,{ }^{2}$ the condensation temperature $T_{c},{ }^{3}$ and the random-phase approximation (RPA) dielectric response function ${ }^{4}$ were studied in the high-density limit, including a two-dimensional Bose gas. ${ }^{5-8}$ In the high-density regime there are many particles within a sphere whose radius is the Bohr radius, the gas parameter $r_{s}=m e^{* 2} /(4 \pi n / 3)^{1 / 3}<<1$.

In this paper we formulate the Bogoliubov-de Gennes (BdG) equations for a charged Bose gas using the Bogoliubov displacement transformation. ${ }^{9}$ We solve these equations for the condensate contribution. The response functions derived are the same as the "free"-boson RPA formula. 4,5 In agreement with "textbook" statements 10 the collective excitations are identical to the singleparticle spectrum.

Our approach differs from the so-called random-phase approximation $^{4,5}$ (RPA) where the one-particle excitations were assumed to behave as free bosons. It also differs from that employed by Ranninger and Thirring ${ }^{11}$ as our result remains gauge invariant when the supracondensate particle response is taken into account.

\section{BOGOLIUBOV APPROACH} TO THE ELECTRODYNAMICS OF CHARGED BOSONS

A system of charged bosons on an oppositely charged background (to ensure charge neutrality) in an external field is described by the Hamiltonian:

$$
\begin{gathered}
H=\int d \mathbf{r} \psi^{\dagger}(\mathbf{r}, t)\left[-\frac{\left(\boldsymbol{\nabla}-i e^{*} \mathbf{A}\right)^{2}}{2 m}+\mu\right] \psi(\mathbf{r}, t) \\
+\frac{1}{2} \int d \mathbf{r} \int d \mathbf{r}^{\prime} V\left(\mathbf{r}-\mathbf{r}^{\prime}\right) \psi^{\dagger}(\mathbf{r}, t) \psi^{\dagger}\left(\mathbf{r}^{\prime}, t\right) \\
\times \psi\left(\mathbf{r}^{\prime}, t\right) \psi(\mathbf{r}, t)
\end{gathered}
$$

with the Fourier component of the Coulomb interaction $V(\mathbf{k})=4 \pi e^{* 2} / k^{2}$ for $3 d$ and zero for $\mathbf{k}=0 . \quad \mathbf{A}(\mathbf{r}, t)$ is the vector potential, $e^{*}$ is the boson charge, $m$ is the boson mass, and $\hbar=c=1$. The equation of motion for the field operator $\psi$ is

$$
\begin{aligned}
i \frac{d}{d t} \psi(\mathbf{r}, t)= & \left.-\frac{\left(\boldsymbol{\nabla}-i e^{*} \mathbf{A}\right)^{2}}{2 m}+\mu\right] \psi(\mathbf{r}, t) \\
& +\int d \mathbf{r}^{\prime} V\left(\mathbf{r}-\mathbf{r}^{\prime}\right) \psi^{\dagger}\left(\mathbf{r}^{\prime}, t\right) \\
& \times \psi\left(\mathbf{r}^{\prime}, t\right) \psi(\mathbf{r}, t) .
\end{aligned}
$$

Using the Bogoliubov displacement transformation ${ }^{9}$ with a space- and time-dependent macroscopic order parameter $\psi_{0}$,

$$
\psi(\mathbf{r}, t)=\psi_{0}(\mathbf{r}, t)+\widetilde{\psi}(\mathbf{r}, t),
$$

and the Hartree approximation for the interaction between bosons pushed up from the condensate, supracondensate bosons $\widetilde{\psi}$, one obtains a set of linear equations of the Bogoliubov-de Gennes type:

$$
\begin{aligned}
i \frac{d}{d t} \psi_{0}(\mathbf{r}, t)= & {\left[-\frac{\left(\nabla-i e^{*} \mathbf{A}\right)^{2}}{2 m}+\mu\right] \psi_{0}(\mathbf{r}, t) } \\
& +\int d \mathbf{r}^{\prime} V\left(\mathbf{r}-\mathbf{r}^{\prime}\right)\left\{n\left(\mathbf{r}^{\prime}, t\right) \psi_{0}(\mathbf{r}, t)+\left\langle\widetilde{\psi}^{\dagger}\left(\mathbf{r}^{\prime}, t\right) \widetilde{\psi}(\mathbf{r}, t)\right\rangle \psi_{0}\left(\mathbf{r}^{\prime}, t\right)+\left\langle\widetilde{\psi}\left(\mathbf{r}^{\prime}, t\right) \widetilde{\psi}(\mathbf{r}, t)\right\rangle \psi_{0}^{*}\left(\mathbf{r}^{\prime}, t\right)\right\}
\end{aligned}
$$

and 


$$
\begin{aligned}
i \frac{d}{d t} \widetilde{\psi}(\mathbf{r}, t)= & \left.-\frac{\left(\boldsymbol{\nabla}-i e^{*} \mathbf{A}\right)^{2}}{2 m}+\mu\right] \widetilde{\psi}(\mathbf{r}, t) \\
& +\int d \mathbf{r}^{\prime} V\left(\mathbf{r}-\mathbf{r}^{\prime}\right)\left\{n\left(\mathbf{r}^{\prime}, t\right) \widetilde{\psi}(\mathbf{r}, t)+\left[\psi_{0}^{*}\left(\mathbf{r}^{\prime}, t\right) \psi_{0}(\mathbf{r}, t)+\left\langle\widetilde{\psi}^{\dagger}\left(\mathbf{r}^{\prime}, t\right) \widetilde{\psi}(\mathbf{r}, t)\right\rangle\right] \widetilde{\psi}\left(\mathbf{r}^{\prime}, t\right)\right. \\
& \left.+\left[\psi_{0}\left(\mathbf{r}^{\prime}, t\right) \psi_{0}(\mathbf{r}, t)+\left\langle\widetilde{\psi}\left(\mathbf{r}^{\prime}, t^{\prime}\right) \widetilde{\psi}(\mathbf{r}, t)\right\rangle\right] \widetilde{\psi}^{\dagger}\left(\mathbf{r}^{\prime}, t^{\prime}\right)\right\}
\end{aligned}
$$

where

$$
n(\mathbf{r}, t)=\left|\psi_{0}(\mathbf{r}, t)\right|^{2}+\left\langle\widetilde{\psi}^{\dagger}(\mathbf{r}, t) \widetilde{\psi}(\mathbf{r}, t)\right\rangle
$$

is the boson density.

In the high-density limit, $r_{s}=m e^{* 2} /(4 \pi n / 3)^{1 / 3}<<1$, the contribution from terms nonlinear in $\widetilde{\psi}$ is small.

Applying a linear transformation for $\widetilde{\psi}$,

$$
\widetilde{\psi}(\mathbf{r}, t)=\sum_{v} u_{v}(\mathbf{r}, t) \alpha_{v}+v_{v}^{*}(\mathbf{r}, t) \alpha_{v}^{\dagger},
$$

where $\alpha_{v}$ and $\alpha_{v}^{\dagger}$ are bosonic quasiparticle operators for the state $v$, and omitting the Hartree terms we obtain from Eq. (5) two coupled Schrödinger equations for $u$ and $v$ :

$$
\begin{aligned}
i \frac{d}{d t} u(\mathbf{r}, t)= & {\left[-\frac{\left(\nabla-i e^{*} \mathbf{A}\right)^{2}}{2 m}+\mu\right] u(\mathbf{r}, t) } \\
& +\int d \mathbf{r}^{\prime} V\left(\mathbf{r}-\mathbf{r}^{\prime}\right)\left\{\left|\psi_{0}\left(\mathbf{r}^{\prime}, t\right)\right|^{2} u(\mathbf{r}, t)+\psi_{0}^{*}\left(\mathbf{r}^{\prime}, t\right) \psi_{0}(\mathbf{r}, t) u\left(\mathbf{r}^{\prime}, t\right)+\psi_{0}\left(\mathbf{r}^{\prime}, t\right) \psi_{0}(\mathbf{r}, t) v\left(\mathbf{r}^{\prime}, t\right)\right\}
\end{aligned}
$$

and

$$
\begin{aligned}
-i \frac{d}{d t} v(\mathbf{r}, t)= & \left.-\frac{\left(\nabla+i e^{*} \mathbf{A}\right)^{2}}{2 m}+\mu\right] v(\mathbf{r}, t) \\
& +\int d \mathbf{r}^{\prime} V\left(\mathbf{r}-\mathbf{r}^{\prime}\right)\left\{\left|\psi_{0}\left(\mathbf{r}^{\prime}, t\right)\right|^{2} v(\mathbf{r}, t)+\psi_{0}\left(\mathbf{r}^{\prime}, t\right) \psi_{0}^{*}(\mathbf{r}, t) v\left(\mathbf{r}^{\prime}, t\right)+\psi_{0}^{*}\left(\mathbf{r}^{\prime}, t\right) \psi_{0}^{*}(\mathbf{r}, t) u\left(\mathbf{r}^{\prime}, t\right)\right\}
\end{aligned}
$$

There is also a constraint to retain the Bose commutation relation for $\widetilde{\psi}$ :

$$
\sum_{v}\left[u_{v}(\mathbf{r}, t) u_{v}^{*}\left(\mathbf{r}^{\prime}, t\right)-v_{v}\left(\mathbf{r}^{\prime}, t\right) v_{v}^{*}(\mathbf{r}, t)\right]=\delta\left(\mathbf{r}-\mathbf{r}^{\prime}\right) .
$$

Equations (8)-(10) together with Eq. (4) describe the electrodynamics of charged bosons, playing the same role as the time-dependent Ginzburg-Landau equations for the BCS superconductor.

\section{ZERO-FIELD SOLUTION}

The $\mathbf{r}$ and $t$ dependences of $u_{\mathbf{k}}(\mathbf{r}, t)$ and $v_{\mathbf{k}}(\mathbf{r}, t)$ for the homogeneous case when $\mathbf{A}=0$ are

$$
u_{\mathbf{k}}(\mathbf{r}, t)=u_{\mathbf{k}} e^{i \mathbf{k} \cdot \mathbf{r}-i \epsilon_{\mathbf{k}} t}
$$

and

$$
v_{\mathbf{k}}(\mathbf{r}, t)=v_{\mathbf{k}} e^{i \mathbf{k} \cdot \mathbf{r}-i \epsilon_{\mathbf{k}} t} .
$$

The condensate macroscopic wave function is time and space independent, $\psi_{0}=\sqrt{n_{0}}$, which gives from Eq. (4)

$$
\mu=0 \text {. }
$$

Substituting Eqs. (11)-(13) into Eqs. (8) and (9), one obtains

$$
\begin{aligned}
& \epsilon_{\mathbf{k}} u_{\mathbf{k}}=\frac{k^{2}}{2 m} u_{\mathbf{k}}+n_{0} V(\mathbf{k})\left\{u_{\mathbf{k}}+v_{\mathbf{k}}\right\}, \\
& -\epsilon_{\mathbf{k}} v_{\mathbf{k}}=\frac{k^{2}}{2 m} v_{\mathbf{k}}+n_{0} V(\mathbf{k})\left\{v_{\mathbf{k}}+u_{\mathbf{k}}\right\},
\end{aligned}
$$

and from Eq. (10)

$$
\left|u_{\mathbf{k}}\right|^{2}-\left|v_{\mathbf{k}}\right|^{2}=1 \text {. }
$$

As a result

$$
\begin{aligned}
& u_{\mathbf{k}}^{2}=\frac{1}{2}\left[1+\frac{\xi_{\mathrm{k}}}{\epsilon_{\mathrm{k}}}\right), \\
& v_{\mathbf{k}}^{2}=-\frac{1}{2}\left[1-\frac{\xi_{\mathrm{k}}}{\epsilon_{\mathrm{k}}}\right),
\end{aligned}
$$

and

$$
u_{\mathbf{k}} v_{\mathbf{k}}=-\frac{V(\mathbf{k}) n_{0}}{2 \epsilon_{\mathbf{k}}},
$$

where $\xi_{\mathbf{k}}=k^{2} / 2 m+V(\mathbf{k}) n_{0}$ and $\epsilon_{\mathbf{k}}$ is the one-particle excitation spectrum,

$$
\begin{aligned}
\epsilon_{\mathbf{k}} & =\sqrt{k^{4} / 4 m^{2}+k^{2} / m V(\mathbf{k}) n_{0}} \\
& =\sqrt{k^{4} / 4 m^{2}+\omega_{p 0}^{2}} .
\end{aligned}
$$


Here $\omega_{p 0}^{2}=4 \pi n_{0} e^{* 2} / m$ is the classical plasma frequency for charged particles of density $n_{0}$. The one-particle excitation spectrum Eq. (20) was obtained by Foldy ${ }^{2}$ using the direct diagonalization of the Hamiltonian.

In the high-density limit the density of supracondensate particles is small, ${ }^{2}$

$$
\widetilde{n}=\left\langle\widetilde{\psi}^{\dagger}(\mathbf{r}, t) \widetilde{\psi}(\mathbf{r}, t)\right\rangle=\sum_{\mathbf{k} \neq 0} v_{\mathbf{k}}^{2} \simeq 0.2 n r_{s}^{3 / 4}
$$

The interaction energy of the supracondensate bosons (the Hartree term) is also small.

\section{THE GROUND-STATE ENERGY}

Foldy also derived the ground-state energy:

$$
E_{0}=\frac{1}{2} \sum_{k \neq 0}\left(\epsilon_{\mathbf{k}}-\xi_{\mathbf{k}}\right)
$$

This can be written in terms of the plasma frequency $\omega_{p 0}$ :

$$
\begin{aligned}
E_{0} & =\frac{2 \sqrt{2}}{3^{1 / 4} \pi} n \omega_{p 0} r_{s}^{3 / 4} \int_{0}^{\infty} d k k^{2}\left[\sqrt{k^{4}+1}-k^{2}-\frac{1}{2 k^{2}}\right) \\
& \simeq-0.23 n \omega_{p 0} r_{s}^{3 / 4} .
\end{aligned}
$$

The negative value of the ground-state energy is a product of the oppositely charged background. This can be shown from a simple argument.

The ground-state energy is estimated as the zero-point energy of supracondensate bosons plus the potential energy arising from statistical fluctuations of the boson density. The zero-point energy is calculated from the kinetic energy of a single supracondensate boson confined to the average volume occupied by one such boson $\left(\sim \tilde{n}^{-1}\right)$. This produces an energy per unit volume of

$$
E_{k}=\frac{\tilde{n}^{5 / 3}}{2 m} \text {. }
$$

From classical thermodynamics the statistical fluctuation of the number of bosons in a given volume goes as the square root of the total number of bosons within that volume. No fluctuations can occur in the oppositely charged background, producing a potential energy associated with these fluctuations. The fluctuation in the number of particles is $\delta n=\sqrt{n / \tilde{n}}$, with $n$ the total density of bosons. This results in a potential energy per unit volume of

$$
E_{p}=-e^{* 2} \delta n^{2} \widetilde{n} \tilde{n}^{1 / 3}=-e^{* 2} n \tilde{n}^{1 / 3} .
$$

The sum of the potential and kinetic energy, Eqs. (24) and (25),

$$
E_{0}=\frac{\widetilde{n}^{5 / 3}}{2 m}-e^{* 2} n \tilde{n}^{1 / 3},
$$

is then minimized with respect to the supracondensate density, $\tilde{n}$, producing the solution

$$
\tilde{n} \simeq 0.72 n r_{s}^{3 / 4} \text {. }
$$

Substituting the value for $\tilde{n}$ into Eq. (26) gives the ground-state energy,

$$
E_{0} \simeq-0.31 n \omega_{p 0} r_{s}^{3 / 4} .
$$

This estimation of the ground-state energy yields the correct exponent of $r_{s}$ and the numerical coefficients are reasonably close to that obtained by Foldy, Eq. (23).

The value of $E_{0}$ is considered as a gain in the total energy due to condensation of interacting bosons with respect to the ground-state energy $(=0)$ of an ideal Bose gas. Therefore $\left|E_{0}\right|$ is the condensation energy.

\section{LINEAR-RESPONSE FUNCTION}

The vector potential affects both the order parameter and the excitation wave functions. To the leading order of $r_{s}$ the equation of motion for the condensate in the presence of a vector potential is

$$
\begin{aligned}
i \frac{d}{d t} \psi_{0}(\mathbf{r}, t)= & -\frac{\left(\boldsymbol{\nabla}-i e^{*} \mathbf{A}\right)^{2}}{2 m} \psi_{0}(\mathbf{r}, t) \\
& +\int d \mathbf{r}^{\prime} V\left(\mathbf{r}-\mathbf{r}^{\prime}\right)\left|\psi_{0}\left(\mathbf{r}^{\prime}, t\right)\right|^{2} \psi_{0}(\mathbf{r}, t) .
\end{aligned}
$$

Using a perturbed wave function,

$$
\psi_{0}(\mathbf{r}, t)=\sqrt{n_{0}}+\phi(\mathbf{r}, t)
$$

and only keeping terms linear in $\mathbf{A}$ one obtains for the Fourier component $\phi(\mathbf{q}, \omega)$,

$$
\begin{aligned}
\omega \phi(\mathbf{q}, \omega)= & \frac{q^{2}}{2 m} \phi(\mathbf{q}, \omega) \\
& +n_{0} V(\mathbf{q})\left\{\phi(\mathbf{q}, \omega)+\phi^{*}(-\mathbf{q},-\omega)\right\} \\
& -\frac{e^{*} \sqrt{n_{0}}}{2 m} \mathbf{q} \cdot \mathbf{a}(\mathbf{q}, \omega) .
\end{aligned}
$$

For a real potential $\mathbf{a}(\mathbf{q}, \omega)=\mathbf{a}^{*}(-\mathbf{q},-\omega)$ and the solution is

$$
\begin{aligned}
& \phi(\mathbf{q}, \omega)+\phi^{*}(-\mathbf{q},-\omega)=-\frac{e^{*} \sqrt{n_{0}}}{m} \frac{\omega}{\omega^{2}-\epsilon_{\mathbf{q}}^{2}} \mathbf{q} \cdot \mathbf{a}(\mathbf{q}, \omega) \\
& \phi(\mathbf{q}, \omega)-\phi^{*}(-\mathbf{q},-\omega)=-\frac{e^{*} \sqrt{n_{0}}}{m} \frac{2 m}{q^{2}} \frac{\epsilon_{\mathbf{q}}^{2}}{\omega^{2}-\epsilon_{\mathbf{q}}^{2}} \mathbf{q} \cdot \mathbf{a}(\mathbf{q}, \omega) .
\end{aligned}
$$

The linear-response function is defined as

$$
J_{m}(\mathbf{q}, \omega)=K^{m n}(\mathbf{q}, \omega) A_{n}(\mathbf{q}, \omega)
$$

where $\mathbf{J}(\mathbf{q}, \omega)$ and $\mathbf{A}(\mathbf{q}, \omega)$ are the Fourier transform of the current and vector potential, respectively. The vector potential $\mathbf{A}$ is defined as an external vector potential, as the internal interactions of the bosons are explicitly taken into account in the Hamiltonian, Eq. (1). The current is defined as the partial differential of the Hamiltonian with respect to the vector potential and in the leading order of $r_{s}$ is given by 


$$
\begin{aligned}
\mathbf{J}(\mathbf{r}, t)= & -\frac{i e^{*}}{2 m}\left[\psi_{0}^{*}(\mathbf{r}, t) \nabla \psi_{0}(\mathbf{r}, t)\right. \\
& \left.-\left(\nabla \psi_{0}^{*}(\mathbf{r}, t)\right) \psi_{0}(\mathbf{r}, t)\right] \\
& -\frac{e^{* 2}}{m}\left|\psi_{0}(\mathbf{r}, t)\right|^{2} A(\mathbf{r}, t) .
\end{aligned}
$$

Again only keeping terms linear in $\mathbf{A}$, the Fourier transform of the current is

$$
\begin{aligned}
\mathbf{J}(\mathbf{q}, \omega)= & \frac{e^{*} \sqrt{n_{0}}}{2 m} \mathbf{q}\left[\phi(\mathbf{q}, \omega)-\phi^{*}(-\mathbf{q},-\omega)\right] \\
& -\frac{e^{* 2} n_{0}}{m} \mathbf{a}(\mathbf{q}, \omega) .
\end{aligned}
$$

Hence

$$
\begin{aligned}
K^{m n}(\mathbf{q}, \omega)=\frac{e^{* 2} n_{0}}{m}\{ & \left\{\delta^{m n} \frac{\omega^{2}}{\epsilon_{\mathrm{q}}^{2}-\omega^{2}}\right. \\
& \left.+\left(q^{m} q^{n}-\delta^{m n} q^{2}\right) \frac{\epsilon_{\mathrm{q}}^{2}}{q^{2}\left[\epsilon_{\mathrm{q}}^{2}-\omega^{2}\right]}\right\}
\end{aligned}
$$

This response function has been split into longitudinal $K_{l} \sim \delta^{m n}$ and transverse $K_{t} \sim\left(q^{m} q^{n}-\delta^{m n} q^{2}\right)$ parts.

The longitudinal response $(\mathbf{D} \| \mathbf{q})$ can be expressed in terms of the external conductivity $\sigma_{\mathrm{ex}}$ :

$$
\mathbf{J}(\mathbf{q}, \omega)=\sigma_{\mathrm{ex}}(\mathbf{q}, \omega) \mathbf{D}(\mathbf{q}, \omega),
$$

where $\mathbf{D}$ is the external electric field and

$$
\sigma_{\mathrm{ex}}(\mathbf{q}, \omega)=\frac{K_{l}(\mathrm{q}, \omega)}{i \omega}=i \frac{\omega_{p 0}^{2} \omega}{4 \pi\left(\omega^{2}-\epsilon_{\mathrm{q}}^{2}\right)} .
$$

The Kubo ${ }^{12}$ conductivity sum rule is satisfied, in this approximation, if one replaces the total density by $n_{0}$,

$$
\int_{0}^{\infty} d \omega \operatorname{Re} \sigma_{\mathrm{ex}}(\omega)=\frac{1}{8} \omega_{p 0}^{2} \sim n_{0} .
$$

From Eq. (37) the conductivity in the transverse electromagnetic field $(\mathbf{D} \perp \mathbf{q})$ is

$$
\sigma_{t}(\mathbf{q}, \omega)=\frac{i}{4 \pi \lambda_{H}^{2} \omega},
$$

where $\lambda_{H}=\sqrt{m / 4 \pi n_{0} e^{* 2}}$ is the London penetration depth. Equation (41) is the same as for the BCS superconductor. In a constant magnetic field the London equation follows from Eq. (37) for $\omega=0$ which was derived for the interacting Bose gas by Ranninger and Thirring, ${ }^{11}$ who applied a generalized perturbation theory with a constant order parameter $\left(=\sqrt{n_{0}}\right)$. While in the leading order of $r_{s}$ their perturbation theory gives the same response function as derived here, Eq. (37), the supracondensate contribution to the current turns out to be non-gauge-invariant. Our approach is based on the gauge-invariant Eqs. (4), (8), and (9). Hence a charged Bose gas is a superconductor as far as the response on the transverse electromagnetic field is concerned.

\section{SCREENING AND COLLECTIVE EXCITATIONS}

The dielectric response function can be derived from the longitudinal part of the response function, Eq. (37), the continuity equation,

$$
\boldsymbol{\nabla} \cdot \mathbf{J}(\mathbf{r}, t)+\frac{\partial}{\partial t} \rho_{\text {in }}(\mathbf{r}, t)=0,
$$

and the Maxwell equation

$$
\nabla \cdot \mathbf{D}(\mathbf{r}, t)=4 \pi \rho_{\mathrm{ex}}(\mathrm{r}, t) .
$$

For the usual definition of the longitudinal response function

$$
\mathbf{J}(\mathbf{q}, \omega)=K_{l}(\mathbf{q}, \omega) \frac{\mathbf{E}(\mathbf{q}, \omega)}{i \omega},
$$

where $\mathbf{E}$ is an internal field, the dielectric function,

$$
\epsilon(\mathbf{q}, \omega)=\frac{\rho_{\mathrm{ex}}(\mathbf{r}, t)}{\rho_{\mathrm{tot}}(\mathbf{r}, t)},
$$

is given by

$$
\epsilon(\mathbf{q}, \omega)=1+4 \pi \frac{K_{l}}{\omega^{2}} .
$$

When this is combined with the response function, Eq. (37), the resulting dielectric function,

$$
\epsilon(\mathbf{q}, \omega)=1-\frac{\omega_{p 0}^{2}}{\omega^{2}-\epsilon_{\mathbf{q}}^{2}},
$$

describes a dielectric-like material $[\epsilon(\mathbf{g} \rightarrow 0, \omega=0)=2]$ with collective excitations $\Omega=\sqrt{\epsilon_{\mathrm{q}}^{2}+\omega_{p 0}^{2}}$. This is an erroneous result, which originates in the double counting of the Coulomb interaction, Fig. 1.

The correct result is obtained when it is remembered that the response function, Eq. (37), is defined for an external field, D. The relationship between the dielectric function and the longitudinal response is now

$$
\frac{1}{\epsilon(\mathbf{q}, \omega)}=1-4 \pi \frac{K_{l}}{\omega^{2}},
$$

which for the condensate response gives

$$
\epsilon(\mathbf{q}, \omega)=1-\frac{\omega_{p 0}^{2}}{\omega^{2}-q^{4} / 4 m^{2}} .
$$

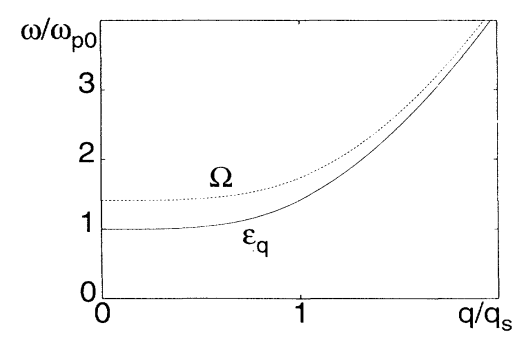

FIG. 1. Excitation spectrum of three-dimensional charged bosons at $T=0$. Dotted line $(\Omega)$ is an artifact of Eq. (42). 
This is the same as the old RPA formula. ${ }^{4,5}$

The screening of the condensate goes as $q^{-4}$, this produces a normalized potential which goes as ${ }^{4}$

$$
\widetilde{V} \sim \frac{e^{-q_{s} r / \sqrt{2}} \cos \left(q_{s} r / \sqrt{2}\right)}{r},
$$

where $q_{s}=\sqrt{2 m \omega_{p 0}}$. This effectively produces a screened potential with a screening radius of $q_{s}$.

The zeros of the dielectric function describe collective excitations of the system, these are the same as the Bogoliubov single-particle excitations,

$$
\Omega=\sqrt{\left(q^{2} / 2 m\right)^{2}+\omega_{p 0}^{2}} .
$$

This is in direct agreement with the old RPA result ${ }^{4,5}$ and textbook statements. ${ }^{10}$

Using the old RPA formalism we are unable to calculate the response of the supracondensate. This is the main advantage of our approach.

\section{SUMMARY}

In summary, taking into account the interaction with the condensate we derive the BdG nonstationary equations and the dielectric response function of threedimensional charged bosons at $T=0$. Despite taking the interaction of the bosons explicitly into account the response function is the same as that for "free" bosons (RPA result). This is a peculiar feature of replacing the condensate propagator with a $c$ number.
In the diagram technique this can be seen as replacing the condensate line with a classical field, representing the condensate. This has the effect of cutting the line, precluding the use of the renormalized supracondensate line in the calculation of the polarization loop with another supracondensate line replaced by a condensate line. If the renormalized supracondensate line is used it results in reducible diagrams for the polarization, and thus double counting of the Coulomb interaction.

There is a direct analogy between excitations of a charged Bose gas and the BCS superconductor. The only difference is that in the BCS superconductor the collective plasmon mode lies well above the BCS quasiparticle energy. In the charged Bose gas the plasmon has an energy identical with the Bogoliubov quasiparticle energy.

Our approach can be easily extended to include the supracondensate particles and their interaction, at least in the Hartree approximation, Eqs. (4) and (5), while remaining gauge invariant. This is contrary to previous perturbation techniques. ${ }^{1.1}$ To include these higher-order terms the space and time dependence of the condensate wave function has to be carefully considered.

\section{ACKNOWLEDGMENTS}

We acknowledge helpful and stimulating discussion with Sir N. F. Mott, A. Gold, D. Khmelnitskii, and J. Wheatley. One of us (A.S.A.) appreciates the financial support from the Leverhulme Trust.
${ }^{1}$ A. S. Alexandrov and N. F. Mott, Supercond. Sci. Technol. 6, 215 (1993).

${ }^{2}$ L. L. Foldy, Phys. Rev. 124, 649 (1961).

${ }^{3}$ R. F. Bishop, J. Low Temp. Phys. 15, 601 (1974).

${ }^{4}$ S. R. Hore and N. E. Frankel, Phys. Rev. B 12, 2619 (1975); 14, 1952 (1976).

${ }^{5}$ D. F. Hines and N. E. Frankel, Phys. Rev. B 20, 972 (1979).

${ }^{6}$ A. Gold, Z. Phys. B 83, 429 (1991).
${ }^{7}$ V. N. Popov, Theor. Math. Phys. 11, 565 (1972).

${ }^{8}$ D. S. Fisher and P. C. Hohenberg, Phys. Rev. B 37, 4936 (1988).

${ }^{9}$ N. Bogoliubov, J. Phys. USSR 11, 23 (1947).

${ }^{10}$ See, for example, D. Pines, The Many-Body Problem (Benjamin/Cummings, Reading, MA, 1961), p. 74.

${ }^{11}$ J. Ranninger and W. Thirring, Z. Phys. 171, 312 (1963).

${ }^{12}$ R. Kubo, J. Phys. Soc. Jpn. 12, 570 (1957). 